\title{
The electrocardiogram characteristics and pacing parameters of Permanent left bundle branch area pacing : A systematic review and meta-analysis
}

\author{
Jia Gao ${ }^{1}$, Bing Zhang ${ }^{1}$, Nan Zhang ${ }^{2}$, Sun Meng ${ }^{2}$, and Wang Rui ${ }^{2}$ \\ ${ }^{1}$ Shanxi Medical University \\ ${ }^{2}$ First Hospital of Shanxi Medical University
}

June 24, 2020

\begin{abstract}
Background : The invention of His bundle pacing technology bring a new revelation-Left bundle branch area pacing (LBBAP). LBBAP engages in the electrical activation through left bundle branch area and produces ventricular electrical synchronization, which can grab more cardiac cells and increase the safety of pacing compared to His bundle pacing (HBP). This pacing has been considered as an attractive mode to achieve normal physiological pace markers. However,as a new technology, LBBAP is still in the stage of clinical exploration and lacks adequate evaluation. Objective :This study aims to investigate the electrocardiogram characteristics,pacing parameters,the safety and the effectiveness of LBBAP. Method : A computerized search of PubMed, Embase, The CochraneLibrary, Chinese CNKI, CBM, Wanfang and Weipu Chinese Sci-tech Journals database for effects of Left bundle pacing. The baseline characteristics, the successful rate of implantation, capture threshold, R-wave amplitude, pacing impedance, QRS duration and follow-up date were extracted and summarized. Results : Thirteen studies including 618 patients were included in this analysis. The overall successful rate for implantation was $92.1 \%$.Permanent LBBAP resulted in narrow QRS duration when patients with QRS duration $\_120 \mathrm{~ms}$ after implantation $(\mathrm{P}=0.02)$. QRS duration and capture threshold of LBBAP remained stable during follow-up. Moreover, there was a trend of increased R-wave amplitude and declined pacing impedance at follow-up compared to implantation $\left(\mathrm{P}=0.003\right.$ and $\mathrm{P}_{j} 0.00001$, respectively). Conclusion : Permanent LBBAP has shown promising results in pacemaker-indicated patients. Good electrical synchronization, high success rates, and stable pacemaker parameters point to the unparalleled advantages of LBBAP in physiological pacing.
\end{abstract}

\section{INTRODUCTION}

cardiac pacing technology as a therapy has been used for over a half century and it remains the most effective therapy for bradycardia and cardiac conduction dysfunction. Traditionally, the pacing site at the apex of the right ventricle has been chosen because of the ease of placement of transvenous pacing leads. Several studies conducted over the past two decades have shown that right ventricular pacing (RVP)has adverse effects on clinical outcomes because of ventricular mechanical asynchrony which is secondary to electrical asynchrony ${ }^{1-3}$. The Pursuit of alternate pacing sites has been widely studied but no definitive conclusion is reached ${ }^{4}$. In 2000, Deshmukh conducted the pioneering investigation of permanent direct His bundle pacing $(\mathrm{HBP})^{5}$. Since then, more and more studies showed that HBP is superior in preserving electrical synchrony and left ventricular(LV)function compared to right ventricular pacing (RVP) ${ }^{6,7}$. However, lead placement for HBP is a technically challenging due to its anatomic location, and long-term capture thresholds of HBP have been found to be significantly higher than those of RVP. In 2017, Huang Weijian proposed left bundle branch area pacing (LBBAP) firstly ${ }^{8}$. Some clinical trials have demonstrated that left bundle branch area pacing exhibited the feasibility for patient with left bundle branch block (LBBB)/heart failure and showed more stable and reliable pacing parameters, but these trials mostly were small sample,short-term 
studies. LBBAP may be a potential physiological pacing method that can replaces HBP, but it lacks of clinical application and requires to investigate the electrocardiogram characteristics, pacing parameters , the safety and the effectiveness .

So we systematically reviewed currently available literature and carried out this meta-analysis: (i) to investigate the electrocardiogram characteristics change (ii)to report the stability of pacing parameters and (iii)to summuerize the successful rate of LBBAP from kinds of articles. The reporting of this systematic review follows current standards.

\section{METHODS}

\subsection{Data sources and searches}

We conducted a systematic search of electronic databases including PubMed, Embase and etc. The search was limited to full-text English or Chinese publications before 31 December 2019. The search terms included "Left bundle branch area " or "LBBAP" and "pacing".

\subsection{Study selection and data abstraction}

The abstracts retrieved from the search were reviewed by two investigators. Articles were included :(1) Study type:observational study and randomized controlled study; (2)study object: patients who need permanent pacemaker implantation; (3)clearly define the conditions for LBBAP success; (4)The ECG characteristics and pacing parameters of baseline and follow-up including QRS duration, capture threshold, R-wave amplitude (perception), impedance, etc. (5)have a clear follow-up time. Articles were excluded: (1) report the acute response only and follow-up time of LBBAP less than 1 month; (2)repeated data from the same research center, unable to obtain full text or original data after contacting the author; (3)case reports, animal studies etc.; (4)documents in languages other than Chinese and English. The extracted data included gender, age, indication of device implantation, number of enrolled patients, success rate of implantation, follow-up time, QRS duration at baseline and follow-up, capture threshold at baseline and follow-up, R-wave amplitude at baseline and follow-up, impedance at baseline and follow-up. For continuous variables, means, standard deviations, and sample sizes were extracted to estimate the overall average values.

\subsection{Statistical analysis}

Review Manager 5.3 (Cochrane, London, UK) was used to perform data analysis using random effects models. Statistical heterogeneity was assessed using the $\mathrm{I}^{2}$ statistic. Sensitivity analyses were conducted to identify potential heterogeneity by leaving out any study. Publication bias was evaluated by generating a funnel plot of the logarithm of effect size against the standard error for each trial.

$\mathrm{P} ; 0.05$ was considered statistically significant in all analyses.

\section{RESULTS}

\subsection{Study characteristics}

Figure 1 shows the results of the search and selection process. Thirteen studies including 618 patients were included in these meta-analyses. The average age of the patients was 69.4 years and 299 of patients were male(48.3\%). The overall successful rate of LBBAP was 92\% (569/618). (Table1)

\subsubsection{QRS duration at baseline and implantation}

For patients with QRS duration $<120 \mathrm{~ms}$, no significant difference was observed between baseline and paced QRS duration ( $107.3 \pm 24.8 \mathrm{~ms}$ vs $115.3 \pm 10.9 \mathrm{~ms}, \mathrm{P}=0.28 \mathrm{I}^{2}=92 \%$,Figure $\left.2 \mathrm{~A}\right)$. For patients with QRS duration $>120 \mathrm{~ms}$, the paced QRS duration was markedly decreased from $140.2+-34.2 \mathrm{~ms}$ at baseline to 133.1 $+-16.7 \mathrm{~ms}$ after LBBAP $\left(\mathrm{P}=0.02, \mathrm{I}^{2}=92 \%\right.$, Figure $\left.2 \mathrm{~B}\right)$, representing a mean narrowing of QRS duration.

\subsubsection{QRS duration after implantation and follow-up}


Despite there are various follow-up time,we adopt the longest follow-up time of every article that we can achieve and all follow-up time $>1$ month at least. Patients accepted LBBAP have stable QRS duration when it compared to implantation ( $\mathrm{P}=0.19, \mathrm{I}^{2}=0 \%$, Figure 3 ).

\subsection{Capture threshold at baseline and follow-up}

Despite the difference in follow-up time, our study did not show the same situation as $\mathrm{HBP}^{6}$, the capture threshold did not increase with longer follow-up time, and there was no statistically significant difference between the postoperative capture threshold and the follow-up threshold ( $\mathrm{P}=0.95, \mathrm{I}^{2}=91 \%$, Figure 4).

\subsection{R-waveamplitude at baseline and follow-up}

After aggregating all studies that recorded R-wave amplitude with relevant follow-up data, we found a significant trend of elevated R-wave amplitude which was statistically significant when comparing the data collected after surgery and at follow-up(12.17+-5.24mv VS $14.22+-5.47 \mathrm{mv}, \mathrm{P}=0.003, \mathrm{I}^{2}=50 \%$, Figure 5).

\subsection{Impedance at baseline and follow-up}

The data revealed a clear trend of decreasing pacing impedance at follow-up and a statistically significant correlation between the postoperative and the follow-up, which may be related to the remission of edema and the formation of fibrous scarring at the electrode fixation site. (684.51+-134.15 $\Omega$ vs $532.15 \pm 104.24 \Omega$ $\mathrm{P} ; 0.00001, \mathrm{I}^{2}=85 \%$, Figure 6 )

\subsection{LBBAP success rate and associated complications}

In the included studies, the highest success rate was $100 \%$, the lowest success rate was $66.7 \%$, and the average success rate was $92 \%$.

It should be noted in particular that the study conducted by author Chen Lu was a discussion of the success rate, safety and efficacy of LBBAP surgery. Their retrospective case study was divided into two distinct phases, namely, the learning phase and the proficient surgery phase, and there was a significant difference in the success rates of the two phases, which were $55.6 \%$ and 88.9 , respectively, with a mean success rate of $66.7 \%$.

In terms of surgery-related complications, one patient in Xiaofei Li's study developed a ventricular septal perforation shortly after surgery and successfully repaired it; In Pugazhendhi Vijayaraman's study, three patients presented with acute lead dislodgments within 24 hours after surgery, and these three patients subsequently underwent surgery and were successfully repaired. In the third patient, another ventricular septal perforation occurred during the re-repair procedure, and the electrode was re-fixed slightly below the previous fixation point. One patient developed a pericardial effusion as a result of uninterrupted oral anticoagulation, which disappeared after puncture drainage; In Yuqiu Li's study, one patient who was receiving dual antiplatelet therapy developed a pockethematoma; In Jingfeng Wang's study, one patient suffered an intraoperative septal lead perforation and three patients developed mild pocket hematoma within one month of surgery, and all hematoma was relieved by reinforced elastic bandage compression. Two patients experienced lead dislodgement. One was noted 2 months post-implant, who received LBBAP again; while another occurred 4 months post-implant with the lead relocated to RVS.

\subsection{Sensitivity analysis}

Sensitivity analysis by leaving out any study did not influence the direction or magnitude of the above results, indicating that no single study overly affected most of the findings. Due to the limited number of included studies for correction threshold, sensitivity analysis might not be suitable in the situation. Funnel plot of the included studies showed that no obvious publication bias was found(Figure 7).

\section{DISCUSSION}

The value of this meta-analysis on LBBAP is that it included almost all types of patients with pacing indications, and self control study showed that LBBAP had a good therapeutic effect. Our meta-analysis 
demonstrated that: (1) LBBAP produces a favorable QRS duration, not only stabilizing patients with a normal QRS duration, but also significantly shortening the prolonged QRS duration; (2)In patients receiving LBBAP, there are the following changes in pacemaker parameters after a period of follow-up: a. the stable capture threshold for a long time; b.a positive trend in R-wave amplitude; c. a negative trend in pacing impedance; (3) In the included studies, the average success rate of LBBAP was high, the overall safety of the procedure was reliable, and there were no specific complications of pacemaker surgery.

It has been confirmed that RV apical pacing was associated with high incidence of heart failure, AF, and mortality due to its abnormal activation propagation and obvious LV contraction delay ${ }^{23-24}$.Other pacing sites just like the septum or outflow tract did not show obvious results in improving clinical outcomes ${ }^{25-26}$.Up to now, His-bundle pacing was considered as the physiological pacing method. However, challenges with His bundle pacing including difficulty in identifying the location of the His bundle, a relative high capture threshold with potential long-term instability, especially in patients who already have cardiac conduction disease such as LBBB, which withheld the widespread application of this pacing ${ }^{6,27}$.

Pacing the LBB that directly capture the left bundle branch by deep septal pacing was first introduced by Huang in $2017^{8}$, which is likely to fill many of the deficits of HBP.The left bundle branch is flat banded and fan-shaped in the subendocardium, with a wider distribution than His bundle and fewer fiber wrapping ${ }^{28,29}$. For the LBBAP procedure, the anatomic characteristics of the LBB provide a relatively large area for the selection of a pacing site. Lead fixation could be more easily achieved by screwing the lead into the ventricular septum. Baseline bundle branch block might be more likely corrected by LBBAP beyond the site of block ${ }^{8,17}$.

The sequence of ventricular excitation and ventricular systolic synchrony are two important factors affecting postoperative cardiac function. The coherence and similarity of the QRS morphology between intrinsic QRS and paced QRS can indirectly reflect the difference of the sequence of downward transmission and ventricular excitation. So the QRS duration is an important indicator of the synchronization of the contractions of both ventricles, which affects the risk of death and heart failure in patients after pacing surgery ${ }^{30-32}$. LBBAP proved its effectiveness both in maintaining a normal QRS duration and in generating narrower QRS duration in our study. The rich myocardial tissue surrounding the electrodes guarantee the characteristic of higher R-wave amplitude with a lower threshold for LBBAP, which was also confirmed in our study. These stable pacing parameters indicated that some sensing or threshold issues that are common in HBP or RVP might be avoided in LBBAP.

In our included study, LBBAP was shown to be effective in the treatment of slow arrhythmias such as AVB and SND. In particular, in patients with HBP failure or CRT indications, LBBAP can maintain better electrical and mechanical synchronization, and its efficacy and safety have been further validated as an excellent alternative to HBP.So it could become a mainstream pacing technology in the future.

\section{STUDY LIMITATION}

There are several limitations of this meta-analysis. First, the number of included patients was limited and most of the studies were cohort studies with inherent limitations that reduced the internal validity compared to randomized controlled trials. Second, there are limited data on the effect size of LBBAP on outcomes as the studies included were observational and did not all have comparative arms. Next, some data, including pacing pulse width and follow-up time, were variable and inconsistent, which may influence the study uniformity. In addition, there was no uniformity at this time in measuring QRS durations with selective and nonselective LBBAP. Finally, the indications for pacemaker placement defined by the studies we included were not uniform and were broad in scope. On the one hand, this demonstrates the great potential and promise of LBBAP for apply in patients with all types of pacemaker indications, and on the other hand, it creates an inevitable heterogeneity in the statistical analysis we perform.

\section{CONCLUSIONS}

Over a certain follow-up time, LBBAP has stable pacing parameters and these indicate a good clinical prognosis. Meanwhile Long-term safety and capture threshold should be continually monitored and awaited 
from further observation. Randomized controlled trials are needed to assess the efficacy of LBBAP.

\section{CONFLICTS OF INTEREST}

The authors declare no conflicts of interest.

\section{FUNDING}

The Cultivation Project of Young and Middle-aged Academic Leaders of the First Hospital of Shanxi Medical University, Grant/Award Number: YD1609.

\section{REFERENCES}

1.Khurshid S, Epstein AE, Verdino RJ, et al. Incidence and predictors of right ventricular pacing-induced cardiomyopathy. Heart Rhythm. 2014;11(9):1619-1625.

2.Hussain MA, Furuya-Kanamori L, Kaye G, Clark J, Doi SA. The Effect of Right Ventricular Apical and Nonapical Pacing on the Short- and Long-Term Changes in Left Ventricular Ejection Fraction: A Systematic Review and Meta-Analysis of Randomized-Controlled Trials. Pacing Clin Electrophysiol. 2015;38(9):11211136.

3.Cho SW, Gwag HB, Hwang JK, et al. Clinical features, predictors, and long-term prognosis of pacinginduced cardiomyopathy. Eur J Heart Fail. 2019;21(5):643-651.

4.Vijayaraman P, Bordachar P, Ellenbogen KA. The Continued Search for Physiological Pacing: Where Are We Now?. J Am Coll Cardiol. 2017;69(25):3099-3114.

5.Deshmukh P, Casavant DA, Romanyshyn M, Anderson K. Permanent, direct His-bundle pacing: a novel approach to cardiac pacing in patients with normal His-Purkinje activation. Circulation. 2000;101(8):869877.

6.Vijayaraman P, Naperkowski A, Subzposh FA, Abdelrahman M, Sharma PS, Oren JW et al. Permanent His-bundle pacing: long-term lead performance and clinical outcomes. Heart Rhythm 2018;15:696-702.

7.Zhang J, Guo J, Hou X, Wang Y, Qian Z, Li K et al. Comparison of the effects of selective and non-selective His bundle pacing on cardiac electrical and mechanical synchrony. Europace 2018;20:1010-7.

8.Huang W, Su L, Wu S, et al. A Novel Pacing Strategy With Low and Stable Output: Pacing the Left Bundle Branch Immediately Beyond the Conduction Block. Can J Cardiol. 2017;33(12):1736.e1-1736.e3.

9.Hou X, Qian Z, Wang Y, et al. Feasibility and cardiac synchrony of permanent left bundle branch pacing through the interventricular septum.Europace. 2019;21(11):1694-1702.

10.Chen K, Li Y, Dai Y, et al. Comparison of electrocardiogram characteristics and pacing parameters between left bundle branch pacing and right ventricular pacing in patients receiving pacemaker therapy. Europace.2019;21(4):673-680.

11.Hasumi E, Fujiu K, Nakanishi K, Komuro I. Impacts of Left Bundle/Peri-Left Bundle Pacing on Left Ventricular Contraction. Circ J.2019;83(9):1965-1967.

12.Zhang W, Huang J, Qi Y, et al. Cardiac resynchronization therapy by left bundle branch area pacing in patients with heart failure and left bundle branch block. Heart Rhythm. 2019;16(12):1783-1790.

13.Li X, Li H, Ma W, et al. Permanent left bundle branch area pacing for atrioventricular block: Feasibility, safety, and acute effect. Heart Rhythm. 2019;16(12):1766-1773.

14.Vijayaraman P, Subzposh FA, Naperkowski A, et al. Prospective evaluation of feasibility and electrophysiologic and echocardiographic characteristics of left bundle branch area pacing. Heart Rhythm. 2019;16(12):1774-1782. 
15.Li Y, Chen K, Dai Y, et al. Left bundle branch pacing for symptomatic bradycardia: Implant success rate, safety, and pacing characteristics. Heart Rhythm. 2019;16(12):1758-1765.

16.Wang J, Liang Y, Wang W, et al. Left bundle branch area pacing is superior to right ventricular septum pacing concerning depolarization-repolarization reserve. J Cardiovasc Electrophysiol. 2020;31(1):313-322.

17.Su L,Wu S,Wang S, Vijayaraman P, Ellenbogen KA, Huang W. Peri-left bundle branch pacing in a patient with right ventricular pacing-induced cardiomyopathy and atrioventricular infra-Hisian block. Europace. 2019;21(7):1038.

18.ZENG Chun-miao, PENG Shuang-lin, RUAN Tian-cheng.Evaluation of the short-term efficacy and safety of left bundle branch pacing in the treatment of patients with atrioventricular blockJ.Journal of Guang Dong Medical University, 2019,37(01):41-44.(Chinese)

19.Chen Lu,Ma Xuexing,Weng Jiayi,He Hongtao,Lu Wenchao,Wang Xi,Xu Guidong,Sun Kangyun,Zou Jiangang.The preliminary application of left bundle branch area pacingJ.Journal of Nanjing Medical University,2019,39(06):818-821.(Chinese)

20.QIAN Zhihong,QIAO Sifen,JIANG Jun,SUN Wei.Efficiency analysis of left bundle branch pacing in treating patients with chronic persistent atrial fibrillation featured by wide QRS wave and heart failureJ.Journal of Clinical Medicine in Practice,2018,22(23):10-12.(Chinese)

21.Ren Cheng,Yao Yunjie,Li Kebei,Wang Li,Sheng Yufeng,Qian Xuesong,Zou Jiangang.Preliminary clinical experience of His-Purkinje system pacingJ.Journal of Nanjing Medical University,2019,39(06):811817.(Chinese)

23.Tops LF, Schalij MJ, Bax JJ. The effects of right ventricular apical pacing on ventricular function and dyssynchrony implications for therapy. J Am Coll Cardiol. 2009,54(9):764-776.

24. Udo EO, van Hemel NM, Zuithoff NP, Doevendans PA, Moons KG. Risk of heart failure- and cardiac death gradually increases with more right ventricular pacing. Int J Cardiol. 2015;185:95-100.

25.Zografos TA, Siontis KC, Jastrzebski M, et al. Apical vs. non-apical right ventricular pacing in cardiac resynchronization therapy: a meta-analysis. Europace. 2015;17(8):1259-1266.

26.Shimony A, Eisenberg MJ, Filion KB, Amit G. Beneficial effects of right ventricular non-apical vs. apical pacing: a systematic review and meta-analysis of randomized-controlled trials. Europace. 2012;14(1):81-91.

27.Huang W, Su L, Wu S et al Long-term outcomes of His bundle pacing in patients with heart failure with left bundle branch block. Heart (2019)105:137-143

28. Anderson RH,Boyett MR,Dobrzynski H,et al. The anatomy of the conduction system:implications for the clinical cardiologistJ.J Cardiovasc Translat Res,2013,6(2):187-196

29.AndersonRH,Beker E, Tranun-Jensen J, Janse MJ Anatomico-electrophysiological correla-tions in the conduction system - a review. BrHeartJ(1981)45:67-82

30.Pang BJ, Kumar S, Tacey MA, Mond HG. Capturing the His-Purkinje system is not possible from conventional right ventricular apical and nonapical pacing sites. Pacing Clin Electrophysiol. 2014;37(6):724730.

31. Turak O, Ozcan F, Canpolat U, et al. Relation between QRS duration and atrial synchronicity in patients with systolic heart failure.Echocardiography. 2014;31(8):972-979.

32.Sinagra G, Proclemer A, Zecchin M. Resynchronization therapy in heart failure: the 'nonresponder'. J Cardiovasc Med (Hagerstown). 2018;19 Suppl 1:e112-e115. 


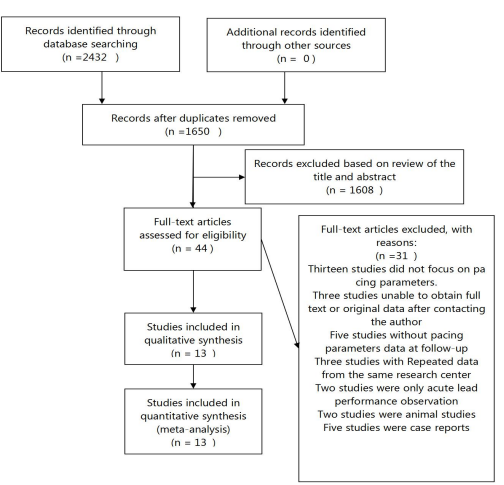

(Figure 1 Flowchart of selection process for articles included in the meta-analysis)

\begin{tabular}{|c|c|c|c|c|c|c|c|}
\hline Authors & Year & Design & Indication & \begin{tabular}{|c|}
$\begin{array}{c}\text { Patient } \\
\text { numeber }\end{array}$ \\
\end{tabular} & \begin{tabular}{|l|} 
successful \\
LBBAP \\
\end{tabular} & \begin{tabular}{|c|}
$\begin{array}{c}\text { Follow-up } \\
\text { time }\end{array}$ \\
\end{tabular} & \begin{tabular}{|c|} 
Implant \\
success(\%)
\end{tabular} \\
\hline Xiaofeng Hou ${ }^{|9|}$ & 2019 & $\begin{array}{c}\text { prospective cohort } \\
\text { study }\end{array}$ & $\begin{array}{l}\text { SND(16) or AVB(21) or AF with slow ventricular } \\
\text { rate(19) }\end{array}$ & 59 & 56 & 1M,.6M & $96 \%$ \\
\hline Keping Chen ${ }^{[10]}$ & 2018 & $\begin{array}{c}\text { prospective cohort } \\
\text { study }\end{array}$ & SND(15) or AVB(1) or RBBB(2) or LBBB(1) & 20 & 20 & зм & $100 \%$ \\
\hline Eriko Hasumi ${ }^{111}$ & 2019 & $\begin{array}{c}\text { prospective cohort } \\
\text { study }\end{array}$ & HBP failed & 21 & 17 & $1 \mathrm{~W}, 3 \mathrm{M}, 6 \mathrm{M}$ & $81 \%$ \\
\hline Weiwei Zhang $^{[12]}$ & 2019 & $\begin{array}{c}\text { prospective cohort } \\
\text { study }\end{array}$ & $\mathrm{HF}$ and LBBB & 11 & 11 & $3 \mathrm{M}, 6 \mathrm{M}, 12 \mathrm{M}$ & $100 \%$ \\
\hline Xiaofei $L^{L^{133}}$ & 2019 & $\begin{array}{l}\text { retrospective } \\
\text { conohit study }\end{array}$ & AVB & 33 & 30 & зм & $90.9 \%$ \\
\hline $\begin{array}{l}\text { Pugazhendhi } \\
\text { Vijayaraman }^{\text {14l }}\end{array}$ & 2019 & $\begin{array}{c}\text { prospective cohort } \\
\text { study }\end{array}$ & $\begin{array}{l}\text { SND(23) or AVBS(54) or CRT(11)or HBP failed(7) } \\
\text { or AV node ablation (7) }\end{array}$ & 100 & 93 & 3м & $93 \%$ \\
\hline Yuqiu Litit1] & 2018 & $\begin{array}{l}\text { prospective cohort } \\
\text { study }\end{array}$ & SND(59) or $A \vee B(28)$ & 87 & 70 & $3 \mathrm{M}$ & $80.4 \%$ \\
\hline Jingfeng Wang ${ }^{160}$ & 2019 & $\begin{array}{c}\text { prospective cohort } \\
\text { study }\end{array}$ & $\begin{array}{l}\text { SND(22) or AVB(36) or AF with slow ventricular } \\
\text { rate(8) }\end{array}$ & 66 & 61 & $1 \mathrm{M}, 3 \mathrm{M}, 6 \mathrm{M}$ & $92 \%$ \\
\hline Su $\operatorname{Lan}^{[17]}$ & 2019 & $\begin{array}{c}\text { prospective cohort } \\
\text { study }\end{array}$ & AVB & 139 & 139 & $\begin{array}{c}2 \mathrm{M}, 3 \mathrm{M}, 6 \mathrm{M}, 1 \\
2 \mathrm{M}\end{array}$ & $100 \%$ \\
\hline ZENG Chun-miao ${ }^{181 \mid}$ & 2019 & $\begin{array}{c}\text { prospective cohort } \\
\text { study }\end{array}$ & AVB(24) or AF with slow ventricular rate(1) & 25 & 25 & $1 \mathrm{~W}, 3 \mathrm{M}, 6 \mathrm{M}$ & $100 \%$ \\
\hline Chen Lu' ${ }^{1291}$ & 2019 & $\begin{array}{l}\text { retrospective } \\
\text { cohort study }\end{array}$ & SND or AVB or AF with slow ventricular rate & 27 & 18 & зм & $66.7 \%$ \\
\hline QIAN Zhihong ${ }^{[20]}$ & 2018 & $\begin{array}{l}\text { retrospective } \\
\text { cohort study }\end{array}$ & AF with slow ventricular rate & 10 & 10 & $\begin{array}{c}7 \mathrm{D}, 30 \mathrm{D}, 3 \mathrm{M}, \\
6 \mathrm{M}\end{array}$ & $100 \%$ \\
\hline Ren Cheng g $21 \mid$ & 2019 & $\begin{array}{l}\text { retrospective } \\
\text { cohort study }\end{array}$ & $\begin{array}{l}\text { SND(6) or AVB (9) or AF with slow ventricular } \\
\text { rate (3) CRT failed(1) pacemaker } \\
\text { reimplantation(1) }\end{array}$ & 20 & 19 & 1W,3M & $95 \%$ \\
\hline
\end{tabular}

Table 1 General description of the included studies 